\title{
Chapter 5 \\ Psychological Grounds \\ of the Development of Ideal Actions \\ and Concepts
}

\section{Outline of Lecture 5}

Galperin starts by reminding that the development of mental actions comprises two parts, orienting and executive, and both are transferred to the learner's mental plane. The orienting partof the action has a guiding function that is needed to perform the action; therefore, the executive part of the action is connected to the orienting part. The orienting part includes four main purposes or tasks: (i) evaluating the present situation; (ii) identifying the potential of the objects present in the situation for meeting the actual needs of the learner; (iii) creating a plan for the action; and (iv) controlling the action's execution according to the created plan. Each of these four tasks or purposes of the action's orienting part can become separate areas of study. Galperin points out that it is important to trace the development of the action's orienting base as well as the changes that this process may undergo during the development of the action. The phases in the development of the action can be used to identify what was overlooked in the learning process and to make the required corrections. Such corrections may include changes to the orienting and executive parts of the action, which are particularly important if (i) a completely new action is developed with learners, and (ii) if an action developed with learners requires corrections.

The development of mental actions begins in the materialised form during learners' interactions with material or materialised resources, followed by the transfer of the action to communicative thinking, where for the first time the action acquires the form of an objective thought. During these transformations, the action is transferred to the learner's mental plane where the action undergoes further changes. These transformations lead to the appearance of a mental phenomenon that is usually studied using two approaches: (i) examining the physiological aspects of the learning process, and (ii) studying the objective logic relationships that are manifest in a thought. Galperin emphasises that in the near future, psychologists may be able to study psychological phenomena by examining the physiological processes in the brain. 
The method of logic allows for studying psychological phenomena only in their final phase, but it does not provide any information about the origin of these phenomena. Galperin argues that researchers should not study phenomena as such, but they should study their ontological grounds as well as the causes and the processes behind these phenomena. He suggests that psychological phenomena should be understood as internal actions that have been transformed from external actions with objects. How the actions are performed indicates whether they have been developed correctly. Therefore, psychologists should study the development of mental actions.

The discussion of the development of mental actions is continued by considering the development of concepts. Galperin indicates that concepts constitute one of the two main forms of reflecting on the real world; the second form is sensory images. The development of concepts is central to learning in schools and higher education.

Previous research examined the development of concepts by learners in schools: scientific concepts and under laboratory conditions, artificial concepts. In school learning, the concept was explained by the teacher, and the learners mastered the characteristic features of the concept by reading relevant texts and solving problems. Under laboratory conditions, the learners identified the characteristic features of the concept by examining a set of objects that encapsulated its features. The results showed that the process of the development of artificial concepts was challenging for learners. These two methods of developing concepts were used in many subject areas and in different countries; however, they had similar mediocre learning outcomes. In both methods, the learners used substantial amounts of time to develop their understanding of the target concepts.

Galperin concludes that first, the development of concepts in learners happens gradually and over time. Second, generalising the target concept also happens over time. By applying the concept in various situations, learners enrich it with features that are derived from their everyday life experiences. However, because such concepts lack structure, the essential (scientific) and inessential (everyday life features) are given equal weight, and students struggle to identify the primary and secondary features of the concept. In addition, the development of conceptual understanding is possible in 11 and 12-year-old students. Galperin suggests that the development of scientific concepts is possible in 6 and 7-year-old students by identifying the characteristic features of the target concept and introducing them to the learners. First, learners must identify the presence or the absence of the characteristic features of the target concept in the object and then determine whether the object belongs to the target concept. Second, the characteristic features of the target concept must be presented to the learners on an orienting card and arranged in a column where each feature is under its number. Third, different tasks should be offered to learners (based on the principle of contrast), such as subject-specific, logical, and psychological tasks. By following this approach, learners develop an understanding of the target scientific concept, and the difference between their achievements becomes insignificant. The learning process happens quickly without memorisation, and the learners are able to apply the target concept in various situations. 


\section{Lecture 5}

I would like to briefly remind you of the content of our previous lectures. We touched upon the subject of psychology, which is of particular importance for contemporary psychology. We argued that psychology should study a person's orienting activity. The orienting part of an action has a guiding function that is needed to perform an action; therefore, the executive part of an action depends on and is inherently connected with the orienting part. Usually the orienting part is developed spontaneously under the influence of some visible final outcomes of the action. Such an approach is insufficient to ensure the action's desired properties. The orienting part of the action carries four main tasks or purposes: (i) evaluation of the present situation, (ii) identifying the potential of the objects present in the situation for the actual needs of the learner, iii) creating a plan of the action and iv) control of the action's execution according to the created plan. Sometimes, the control of the action's execution may turn into an evaluation of our understanding of the present situation.

The four tasks or purposes of the action's orienting part are quite straightforward, though sometimes each of these tasks can turn into separate areas of study. For example, what we call evaluation of the present situation can turn into an investigation of the surrounding world and a human being as a part of this world. Such an investigation can be divided into several independent areas. This is an example of how one part of the orienting activity can turn into several independent areas of study.

A similar situation is when we create a plan. Sometimes creating a plan can turn, as for example, with animals, into a study of an action's trajectory from its start to its final point. It is absolutely necessary to single out this trajectory, and to sketch it so that it can be turned into a sequence of necessary activities. Each of these activities can take different forms but there are always four main tasks (mentioned above) that ultimately ensure a successful action performance.

It is very important to trace in an expanded form the development of an action's orienting base, and what changes this process may undergo during the development of the action. We have traced specifically the process of the development of mental actions. We did so because this process comprises two parts, orienting and executive, and both are transferred to the learner's mental plane, where the action undergoes changes and acquires certain properties. If we are aware of the development process of an action and the changes the action undergoes in its various phases, we can use this action's development phases to identify what has been overlooked to make the required corrections. Such corrections may include changes to the orienting and the executive parts of the action, which may have been created spontaneously or under insufficient control. This is particularly important if (i) this is a completely new action, because following the phases of an action's development can ensure the development of a new action with the desired properties, and (ii) if we need to make some corrections to the action that had been developed incorrectly by learners.

I would like to touch upon an extremely important statement: we begin the development of mental actions with the process of interaction with material or materialised 
objects-materialised action. Materialised action implies interactions with models, signs and diagrams, which means interactions with materialised objects. For example, if we deal with mathematical signs, they can be transferred from one part of a mathematical equation to another, as these signs can be summed up or crossed out-we can interact with these signs like with material objects. You can also check your answer to the problem by performing certain operations with signs. Therefore, we always begin to develop a new action in a materialised or material form and then the action is transferred to communicative thinking, where for the first time the action acquires the form of an objective thought. By going through these phases, the action can be transferred to the learner's mental plane, where the action undergoes further changes. These sequential transformations reflect how an action with material or materialised objects may turn into a thought about this action, though this thought cannot be easily expressed in words. However, it is important that in the cause of these transformations there appears a mental phenomenon. If we were not aware of the process that leads to this phenomenon (mental action), it would be impossible to study the phenomenon of a mental action using objective methods. The question then arises of how researchers can study a mental action. They do so usually in two ways: either by examining physiological aspects of learning processes, or by studying the objective logic relationships that manifest in a thought.

These two methods of studying the learning process are quite common in modern psychology. It is considered that psychological phenomena cannot be subjects of science, as they can only be described. Further, this description is never objective; it is rather an experience which can barely be expressed in words. Therefore, we have a phenomenon, but how can we study it? It would be quite sensible to approach this phenomenon (mental action) by studying the processes that happen in the brain. It might happen that in fifty years, scientists will discover how to trace the processes that happen in the brain. This is a valid method and most psychologists pursue this method to study psychological phenomena. Other scientists approach psychological phenomena by using the logic method. If we were not aware of the development phases of mental actions, we would not have a choice other than to pursue the method of logic, since we can observe the development process of mental actions only in its final phase and we do not know its origin. However, science does not and should not study phenomena, but the causes behind these phenomena, the processes that lead to the observed phenomena.

Yet, what causes the phenomenon of a mental action? As I have already said, if we were not aware of the development phases of mental actions, we would think that mental actions were caused by physiological processes and, therefore, these physiological processes have to be carefully examined to develop our understanding of mental actions. However, we are aware that we are dealing with an internal action that has been transformed from an external action with objects. We have developed this action and make use of it in various situations. We need to control the flow of this action and, in fact, we can sense whether the action is being performed properly. From studies on the physiology of the process of control, we know that this sense appears by comparing the actual flow of the action with the action scheme that we have created. If we get a sense that the actual action flow corresponds to the created 
action scheme, this means that we should not interfere in the action and everything is going well. If we get a sense that the actual action flow does not correspond to the created action scheme, then we should pause the action and look for mistakes. If the correct action has been developed with learners, then the whole action is performed automatically. Therefore, how the action is performed indicates whether the action has been developed correctly and we get this indication by examining the correspondence of the action flow with the created action plan. This makes us consider that it is not a physiological process, but an action that has been developed with learners that lies behind the phenomenon of mental action. The physiological processes may actually assist the performance of the mental action. If a person is ill or tired, then the physiological processes can hinder the action's performance. Therefore, psychology should study the development of mental actions, and not physiological processes. This is an extremely important point related to the main subject of psychology.

\section{Development of Concepts}

The topic development of concepts logically continues the topic development of mental actions, which we have just talked about. Concepts constitute one of the two main forms of reflection of the real world, with sensory images as the second form. We will start with the process of the development of concepts, because concepts are more concrete and, to some extent, easier to understand than sensory images.

All sciences study certain concepts, and developing an understanding of these concepts is one of the most important tasks of the learning process in primary, secondary and higher education. The development of concepts with learners is considered an important characteristic of learners' mental development and we are very much interested in the age when students are able to engage in learning different concepts. In addition, we are interested in the structure of the learning process aimed at the development of concepts with learners.

Studies that examine the development of concepts with learners pursued two approaches: examining the development of concepts during school learning, and of so-called artificial concepts in laboratory conditions. The process of the development of artificial concepts was examined to avoid the influence of the experience concerned with learning in school. In school learning the teacher explains the concept's content to the learners in an appropriate and understandable way, then the learners master the characteristic features of this concept by reading relevant texts in the suggested textbook, and by applying the concept to solve various problems. In laboratory conditions, the following way of learning was pursued: the learners were offered a set of objects that had to be combined in a concept according to their common characteristic features. The combination of these common features was defined by an artificial concept. In doing so, the researchers wanted to trace the development process of concepts with learners. It turned out that it was quite complicated for the learners to create an artificial concept.

These two methods of examining the development process of concepts were completely different, because under the laboratory conditions, the learners had to develop their understanding about the examined objects, and formulate a concept that 
would combine the characteristic features of the target objects. In school learning, the concept was explained to the learners by the teacher to be memorised and applied to solve various problems. It seemed that these two means of developing concepts with learners completely differed from each other. However, these approaches' results appeared to be similar in different subject areas: specifically, in mathematics, geography, history and grammar. In addition, these approaches to studying concepts were similar in different countries and even on different continents. What was similar in these approaches to the development of conceptual understanding was that the achieved learning outcomes were quite mediocre. This was particularly true for learning school concepts, the content of which was presented to the learners at the beginning of the learning process and repeated by the students for several years. It turned out that only after 5-7 years of studying the same concepts the learners developed their understanding about the concepts that were explained to them many years earlier. The students slowly developed their understandings about the characteristic features of the target concept. A similarly slow development of concept happened under laboratory conditions: the learners were able to develop their understandings about one or two characteristic features of the target concept at a time, and then after some time the learners developed their understanding about other characteristic features of the concept, despite that all characteristic features of the target concept were presented to the students at the very beginning of the learning process several years earlier.

Therefore, first, we can conclude that the development of concepts with learners happens gradually over time. Second, generalising a target concept with learners also happens gradually and the learners over time become able to apply the target concept in various situations. It is interesting that in the process of the development of conceptual understanding, learners enrich the concept with other characteristic features that come from their everyday experiences in interacting with the world. Therefore, for a long time, learners might maintain a so-called hybrid understanding of a concept that comprises scientific characteristic features and the features that come from their everyday life experiences. For some time, psychologists admired such a combination of scientific and everyday life features in the target concept that learners maintained, because they considered this an individual approach to learning. However, the presence of the features that stem from everyday life experiences in the learners' understanding of the target concept implies that the concept is lacking structure, and the essential (scientific) and inessential (everyday life features) are given equal weight. Such an understanding on the part of the learners results in the absence of a feature hierarchy, and the inability of the students to identify the target concept's primary and secondary features. Similar results were reported in several studies at different times and in different subject areas; it was therefore concluded that such a process of the development of concepts with learners is inevitable and should be adopted in education.

For a long time, it was considered that the development of conceptual understanding is only possible with 11- and 12-year-old students. First, such an understanding resulted in the idea that learning concepts before the age of 11/12 was not recommended and even forbidden. It was considered that children develop so-called 
quasi- and pseudo-concepts, as they are unable to develop their true understanding of the target concept. Therefore, teachers must introduce incomplete concepts, or a "simplified" version of a concept, and when the children reach the age of 11/12 years old they are able to develop their true understanding of the target concept. On the other hand, why do children of exactly $11 / 12$ years old become able to develop their conceptual understanding? This is the age of adolescence and some think that there is a direct connection between this age and the ability to develop conceptual understanding. This suggestion has had consequences for the practical organisation of the learning process, and it was almost impossible to make changes to such a learning process until we developed the method involving the phases of the development of concepts with learners.

We approached the process of the development of concepts by considering two actions: first, a mental action is only one constituent part of the process of the development of conceptual understanding, and second is the images of objects and the meanings they carry. The students develop their understanding of these meanings when interacting with these objects. Therefore, we need to shift our focus from the development of actions to the development of the images of the objects learners interact with. We have approached this issue in this way: every concept consists of distinctive characteristic features and these features must be identified and introduced to the learners.

First, the learners must identify the presence or the absence of conceptually distinct features in the object they interact with and determine if the object belongs to the target concept. The characteristic features identified in the object must be excessive and necessary to conclude that the object belongs to the target concept. There should not be any other features that the learners simply memorise and do not use. Sometimes, in mathematical concepts studied in school, learners are introduced to features they cannot use. The question then arises, why are these features introduced to the learners? Just to complicate the learning process? We do not accept such general knowledge, and instead select such characteristic features of the target concept that learners are able to use for solving problems.

Second, the characteristic features of the concept must be presented to the learners on the orienting card arranged in a column, each characteristic feature under its number. This is not an easy thing to do, because very often teachers want to explain the target concept in a comprehensive way by introducing the concept features that the learners are not going to use. This should not happen. In addition, we select different types of tasks (subject-specific tasks, logical-type tasks and psychologicaltype tasks) to be solved by the learners. ${ }^{1}$ The order in which these tasks are presented to the learners has to be carefully thought through.

The tasks should be selected based on a psychological principle which was first suggested by Pavlov and discovered in his experiments with dogs. This is the principle of contrast. For example, learners can first be presented with a very easy task, followed by a very difficult task that will enhance full attention on the part of the learner. The teacher presents the characteristic features of the target concept necessary to solve

\footnotetext{
${ }^{1}$ Galperin presents these types of tasks in detail in Lecture 3.
} 
this task. At this point, the orienting card with the characteristic features of the concept is available to the learner. When solving the next tasks, the orienting card is removed, and the learner repeats aloud the characteristic features of the target concept when approaching the task. When solving other tasks, the teacher might indicate the number of characteristic features of the target concept that the student needs to use to solve the task. This means that the learner does not need to repeat all characteristic features of the target concept but solve the task mentally. In doing so, we control the internal learning process of the student. Further in the process, the teacher introduces a task and the learner solves the task mentally, then presents the result to the teacher. Such an approach to the development of concepts with learners, produced totally unexpected results.

First, it turned out that the school concepts that were intended to be developed with 11/12-year-old students, could be developed with children of 7 and even 6 years old. In addition, it turned out that all students in the class developed their understandings of the target concepts. When the concepts are introduced to the learners through traditional teaching, there is always a large spectrum of achievements among the learners: some students learn very fast, other students learn very slowly and some students do not succeed in the learning process at all. To summarise, in a traditional school teaching, we observe different learners: from those who learn fast and easily, to those who struggle and fail in their learning process. Such a difference among the learners' achievements is often explained by the students' different abilities, which seems a reasonable explanation. However, in our approach, we discovered almost no difference between the students' achievements. Of course, some students learned faster than others, but all students developed their conceptual understandings and the difference between the learning outcomes of the individual students was truly insignificant. In traditional school teaching, different learning outcomes are evaluated by marks awarded to the students and, usually, the range of marks in one class is quite wide. When we applied the phases of the development of concepts, we did not observe such a difference between the students' learning outcomes. The learners followed the instructions on the orienting card and they could not miss a single characteristic feature of a target concept when solving a task. In doing so, the learners did not develop any hybrid concepts, though true scientific concepts were developed with the learners which contained the whole set of characteristic features. The learning process happened fast, and the students did not need to memorise the target material. The essential features of the target concept were available for the learners on the orienting card and they could use the orienting card until they did not feel the need to use it when solving the tasks. Only then was the orienting card removed from the learners. The learners developed their understandings of the target concept, and they became able to recall this concept not orally, but to use it to solve various problems.

In traditional school teaching, the learning process is not visible to the learners: it happens in the heads of the individual learners and, therefore, teachers do not have access to this process and cannot control it. For example, a teacher explains the concept, and then checks how the students have understood this concept by asking questions. The students respond to these questions usually by recalling the 
content of the teacher's explanation. Then, the students memorise the content of the concept, but how this happens remains out of the teacher's sight, so the teacher cannot control the learning process. In the next lesson, the teacher checks the students' understanding and it usually turns out that the students demonstrate quite a wide spectrum of understanding of the target concept, which is often explained by the students' different abilities. However, the learning process that led to the development of the students' conceptual understanding was totally uncontrolled and the students were confused by applying both scientific and their everyday experience features of the target concepts. If we create a more rigid framework around this process, it begins to flow quite differently. We do not attempt to explain how the age of adolescence is linked with an ability to develop conceptual understanding, but we are convinced that the students' achievements depend on how the learning process is structured and organised.

In traditional teaching, a new concept is first explained, then memorised, then applied for solving typical problems. After that the concept is applied by learners in other situations. If teachers are unaware of any other methodologies, then such an approach seems quite reasonable, so we should not develop a totally neglectful attitude towards traditional teaching methods. First, the teacher explains a new concept and to be able to apply it in various situations, the learners must develop their understanding of this concept. To achieve this, the learners have to memorise the concept, and then engage in solving various tasks that apply the target concept. This is a logical way to go, but reality, as we know, does not always correspond to the laws of logic.

In our method, students do not need to memorise the target concepts; however, they remember the essential features of these concepts involuntarily. In addition, if the teacher explains the characteristic features of the target concept presented on the orienting card to the learners, and elaborates on how to engage in the learning process by using the orienting card, then the information presented in the orienting card will be memorised very quickly, in 1-1.5 h, and the students may continue solving other problems without the orienting card. Learning happens quickly and without any special efforts from students.

Several researchers are very cautious about such involuntary memorising. American scientists call such memorisation accidental, meaning that it happens by itself, without any effort on the part of the learners. However, we cannot structure the whole learning process on accidental, uncontrolled and chaotic processes. In our method, we can enhance the learners' involuntary memorisation by presenting the characteristic features of the target concepts on the orienting cards, and by having the learners use these orienting cards to solve various problems.

Now, a few words about the outcomes of the learning process that follows the phases of the development of mental actions. Several studies reported that after learners had been exposed to learning through phases, when they encountered a new material or object, they could identify if this material or object belonged to the target concept. For example, a learner comes across a noun in a sentence. The learner sees the word and he is able to identify that it is a noun, and thus demonstrate his conceptual understanding. This phenomenon has been described previously; however, the 
learning process that has led to such a phenomenon happened spontaneously, without interference on the part of the teacher. How does a student identify that the word he/she has come across is a noun? The learner follows the process of identifying the essential characteristic features in the examined object (a word in our case) and concludes that the examined word belongs to the noun concept. When we systematically examine an object that belongs or does not belong to the target concept, we identify a set of features that comprise a so-called dynamic stereotype model. This dynamic stereotype model was first introduced by Pavlov, who suggested that it can imply a different order of the concept's characteristic features. For example, the learner identifies a feature that was presented in the middle of the list of all characteristic features on the orienting card. The dynamic stereotype model is then triggered, and the learner identifies that the examined object belongs to the target concept. However, in this case, a learner can express his understanding by using such words as it seems that this word is a noun. In the later learning process, the student develops his ability to identify all essential characteristic features and conclude that the examined object belongs to the target concept. The learner develops such an ability by interacting with the objects that encapsulate the essential characteristics of the target concepts.

Open Access This chapter is licensed under the terms of the Creative Commons Attribution 4.0 International License (http://creativecommons.org/licenses/by/4.0/), which permits use, sharing, adaptation, distribution and reproduction in any medium or format, as long as you give appropriate credit to the original author(s) and the source, provide a link to the Creative Commons license and indicate if changes were made.

The images or other third party material in this chapter are included in the chapter's Creative Commons license, unless indicated otherwise in a credit line to the material. If material is not included in the chapter's Creative Commons license and your intended use is not permitted by statutory regulation or exceeds the permitted use, you will need to obtain permission directly from the copyright holder. 